Fritz et al.

\title{
Probing Single Biomolecules with Atomic Force Microscopy
}

\author{
JÜRGEN FRITZ* AND DARIO ANSELMETTI \\ Novartis Services AG, Scientific Services, Physics, CH-4002 Basel, Switzerland \\ *also affiliated with: Institute of Physics, University of Basel, Switzerland
}

JANINA JARCHOW AND XAVIER FERNANDEZ-BUSQUETS

Friedrich Miescher-Institute (FMI), CH-4002 Basel, Switzerland

Received January 17, 1997, and in revised form April 26, 1997

\begin{abstract}
During the last years, atomic force microscopy (AFM) has developed from a microscopy tool for solid state surface science towards a method employed in many scientific disciplines such as biology to investigate individual molecules on a nanometer scale. This article describes the current status of the imaging possibilities of AFM on RNA, IgG and gold- labelled cell adhesion molecules, as well as of measurements of intermolecular binding forces between biomolecules in order to investigate their molecular structure, function and elasticity.
\end{abstract}

\section{INTRODUCTION}

The ability of atomic force microscopy (AFM) to image, address and probe single molecules opens fascinating possibilities in order to observe, handle and manipulate individual molecules.

For the investigation of biological materials, to date, AFM is mostly used in the imaging mode of operation under ambient as well as under physiological conditions (Hansma and Hoh, 1994; Bustamante and Keller, 1995). The concept to raster scan a very sharp mechanical sensor at very small distances (typically some $\mathrm{nm}$ ) over a flat surface, on which the molecules of interest have been immobilized, was found not being restricted to hardly any experimental environment. Therefore, by using taylored sample preparation techniques, the immobilized biological molecules can be probed close to their native state.

During the last three years, first attempts have been made to further develop AFM as a local method to measure specific biological recognition forces, in addition to other concepts of force measuring techniques, such as the surface force apparatus (Israelachvili, 1992; Leckband, 1995), and techniques employing pipette suction (Evans et al., 1991), laser tweezers (Svoboda et al., 1993), magnetic beads and hydrodynamic drag (Smith et al., 1992). In the case of AFM, the sharp mechanical sensor has to be functionalized with molecules, which can probe an activated surface carrying their corresponding binding partners.

In this article, we will put our emphasis on both, the imaging capabilities of AFM on different biological molecules such as single stranded RNA (ssRNA), IgG-antibodies (IgG), and gold- labelled cell adhesion molecules, and its capability to measure intermolecular forces and molecular elasticity. 


\section{MATERIALS AND EXPERIMENTAL}

We used a Nanoscope III Multimode AFM from Digital Instruments (DI). Imaging was carried out in the AFM-tapping mode of operation with standard Si-cantilevers (Nanoprobe, Wetzlar). Interaction measurements were done in liquids with a dedicated AFM liquid cell and standard $\mathrm{Si}_{3} \mathrm{~N}_{4}$-cantilevers of a nominal spring constant of $0.06 \mathrm{~N} / \mathrm{m}$ (DI). The exact spring constants were calculated according to the formula described by Neumeister and Ducker (1994). In order to prevent sample damage and unwanted pressure effects in AFM interaction experiments, the maximum repulsive contact force was limited to $500 \mathrm{pN}$. The approach- retract cycles were carried out at typical $\mathrm{z}$-scan rates of $0.1 \mathrm{~Hz}$ to $2 \mathrm{~Hz}$.

Samples for imaging experiments:

$R N A$ : A solution of $3 \mu \mathrm{l}$ RNA $\left(0.1 \mathrm{mg} / \mathrm{ml}\right.$ in $\mathrm{H}_{2} \mathrm{O}$, RNA Length Standard I, Boehringer Mannheim), $30 \mu \mathrm{l} \mathrm{H}_{2} \mathrm{O}$, and $15 \mu \mathrm{l}$ formamide (Fluka) was heated to $50{ }^{\circ} \mathrm{C}$ for some minutes. Then $5 \mu$ l of the solution were put on a mica sheet, which was silanized with aminopropyl-dimethyl-ethoxysilane in the gas phase at room temperature. After 15 minutes the sample was rinsed with water and dried in air prior to imaging.

$I g G$ : Rabbit $\mathrm{IgG}$ solution $(1 \mathrm{mg} / \mathrm{ml}$ in $\mathrm{PBS} \mathrm{pH} 7$, Sigma Chemicals) were put on a dialysis membrane $(0.025 \mu \mathrm{m}$, Millipore) swimming on nanopure water. After 20 minutes $5 \mu$ of the desalted solution were placed on freshly cleaved mica and dried in air.

Gold-labelled MAF: AntiMAF directed antibodies $\alpha 17$ (Block 2 (Misevic et al., 1993), $0.04 \mathrm{mg} / \mathrm{ml}$ in PBS $\mathrm{pH} 7$ ) were mixed with MAF $(0.03 \mathrm{mg} / \mathrm{ml}$ in seawater buffer $)$ in solution. After one hour $5 \mathrm{~nm}$ gold conjugate functionalized with antimouse-IgG (Sigma Chemicals) were added. After another hour $5 \mu \mathrm{l}$ of the solution were put on an ethanol cleaned standard cover slip for $15 \mathrm{~min}$. The glass was rinsed with water and dried in air.

Samples for force-distance measurements::

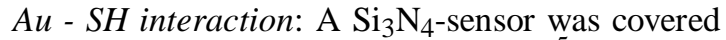
with $3 \mathrm{~nm} \mathrm{Cr}$ and $30 \mathrm{~nm}$ gold at $10^{-5}$ mbar and room temperature. A cover slip was etched for 20 minutes in concentrated $\mathrm{H}_{2} \mathrm{SO}_{4}$ and silanized afterwards with mercaptomethyldimethylethoxysilane (ABCR $\mathrm{GmbH}$, Karlsruhe, Germany) in the gas phase at $50^{\circ} \mathrm{C}$. The measurements were done in nanopure water.

Biotin - avidin interaction: A mercaptosilanized cover slip and a mercaptosilanized $\mathrm{Si}_{3} \mathrm{~N}_{4}$-sensor (silanization see above) were incubated with biotinylated BSA (2 mg/ml in PBS pH 7, Sigma Chemicals) for 2 hours. Afterwards the cover slip was incubated with avidin $(2 \mathrm{mg} / \mathrm{ml}$ in PBS $\mathrm{pH} 7$,
Sigma Chemicals) for 2 hours. The measurements were carried out in PBS at $\mathrm{pH} 7$.

$M A F$ - MAF interaction: A mercaptosilanized cover slip and a mercaptosilanized $\mathrm{Si}_{3} \mathrm{~N}_{4}$-sensor (silanization see above) were incubated for 2 hours with avidin $(2 \mathrm{mg} / \mathrm{ml}$ in PBS $\mathrm{pH} 7$, Boehringer Mannheim), then incubated for 2 hours with biotinylated MAF (1 $\mathrm{mg} / \mathrm{ml}$ in Tris-buffered seawater) and finally saturated with free biotin (1 $\mathrm{mg} / \mathrm{ml}$ in Tris-buffered seawater, Boehringer Mannheim). Measurements were done in a $10 \mathrm{mM}$ $\mathrm{Ca}^{2+}$-containing Tris-buffered seawater at $\mathrm{pH} 7$.

\section{RESULTS AND DISCUSSION}

For imaging biomolecules by AFM, a proper immobilization of the molecules onto a flat surface has to be concepted. For that, in general, four different methods are possible: 1) drying of the sample solution on a surface, 2) physical binding of the molecules, e.g. via counter ions or hydrophobic interaction, to a surface (Thundat et al., 1992; Hansma et al., 1993), 3) chemical binding or crosslinking of the molecules to a surface (Karrasch et al., 1993; Wagner et al., 1996), or 4) embedding of the molecular species in a 2D array (Schabert et al., 1995). In our imaging experiments we mainly used the first method, which is only applicable and renders satisfying results if the system of interest can be imaged under ambient conditions and if the sample preparation process allows removing of the remaining salt of the buffer solution.

As an example, Fig. 1a shows a ssRNA fragment of $5.0 \mathrm{~kb}$, which was immobilized on silanized mica (see preparation) and imaged in air. Its contour length was determined to be about $1300 \mathrm{~nm}$, indicating a length of a single base of about $0.26 \mathrm{~nm}$. The self-affinity of the ssRNA molecules in water induces a more or less globular structure, as can be seen in the inset of Fig. 1a. Therefore, to observe their chain-like structure, a denaturation of the ssRNA fragments, here with formamide, prior immobilization was necessary.

In order to prepare IgGs on an untreated mica surface an additional desalting step before their immobilization was introduced (see preparation). Fig. 1b clearly shows individual isotropically oriented IgGs with their characteristic Y- or heart-shaped structure. That allows an estimate of an actual AFM resolution in the order of 3-5 $\mathrm{nm}$. This resolution seems to be a quite universal value 
for everyday experiments on single immobilized macromolecules measured in the AFM dynamic force- or tapping mode of operation with standard Si-sensors.

As a third example, an AFM immunogold labelling experiment with cell aggregation factors of the marine sponge Microciona prolifera (MAF), a proteoglycan system responsible for cell-cell adhesion, is shown. AntiMAF-directed antibodies $(\mathrm{Ab})$ were bound to MAFs in solution and were labelled with 5 nm gold beads via a secondary antibody. The complex was imaged on a standard cover slip (see preparation). In Fig. 1c, the typical filigran structure of two MAF macromolecules with their central ring structure and 10-15 emanating arms with a length of about $150 \mathrm{~nm}$ can be identified. Superimposed to this structure, monodisperse gold beads are visible as bright spots, partly attached to the ring structure, as well as to the arms of the MAF. Due to the unspecific background of unbound gold beads, a direct localization of the $\mathrm{Ab}$ binding sites on the MAF structure was not possible. However, in Fig. 1d, a larger section of the sample surface clearly exhibited two differently labelled types of MAFs, one obviously with a high affinity and the other with a low or no affinity to the $\mathrm{Ab}$. It was surprising to see, that the sample volume, which was purified from a single sponge individual, consists of two different classes of MAFs.

The last example should also illustrate the problem that when using a single molecule detection method, one always has to be aware of the statistical uncertainty that a probed molecule does not belong to a representative ensemble of the sample volume.

In the following section, we will focus on the investigation of forces between biomolecules by AFM. Upon functionalizing the mechanical sensor of an AFM with a molecule and on functionalizing a surface with the specific binding partner of the molecule, the adhesion force between the sensor and the surface can directly be related to the force between the two binding partners. This AFM mode for measuring adhesion forces is called forcedistance mode, approach-retract cycle or, in general, interaction measurement (Meyer et al., 1988; Weisenhorn et al., 1992): The AFM force sensor is approached towards and consecutively withdrawn from a surface, while the forces acting on the sensor are continously monitored (see Fig. 2 and 3). The adhesion force, and hence the specific biological interaction force, can then be extracted from the force hysteresis in the retracting branch. In order to probe the molecules in their functional state, these experiments have always to be performed in-situ under controlled physiological conditions.

Before starting such measurements, two important questions have to be addressed: First the immobilization of the molecules on sensor and surface has to be strong enough to withstand the expected maximum binding forces. Since molecular interaction forces can be in the order of some hundred $\mathrm{pN}$, only one strong or multiple chemical or biological binding (e.g. a $\mathrm{Au}$ - thiol or a biotin - avidin interaction) allow a proper fixation of the molecules. Thereby, the molecules have to be immobilized oriented or via a flexible crosslinker to guarantee good accessibility to their binding sites. Secondly, in order to discriminate from undesired interactions between sensor and surface, the specificity of the binding has to be confirmed. This can elegantly be done by specifically blocking only the desired interaction and by verifying that no other significant interaction contribute to the measured force-distance curves. The blocking can be realized by adding free binding partners or specific blocking antibodies to the solution, by varying the ion concentration of the buffer (e.g. for the $\mathrm{Ca}^{2+}$-specific MAF - MAF binding), or by using an inactive binding partner for a negative control.

To date, AFM force-distance measurements are reported between members of the biotin - avidin family (Florin et al., 1994; Moy et al., 1994; Lee et al., 1994b; Dammer et al., 1995a; Chilkoti et al., 1995), between complementary oligonucleotides (Lee et al., 1994a; Florin et al., 1995), between cell adhesion molecules (Dammer et al., 1995b), and of different antigen - antibody systems (Dammer et al., 1996; Hinterdorfer et al., 1996). In principle, four different parameters can directly be extracted from force-distance measurements: 1 ) The forces of a single binding event, which is typically in the order of $50 \mathrm{pN}$ between MAFs, antigen - antibodies or small oligonucleotides, and of some hundred $\mathrm{pN}$ for the avidin - biotin or streptavidin - biotin interaction (To account for multiple binding events and for a reasonable 
statistics, one has always to analyse some hundred force-distance curves). 2) The distance between sensor and surface, at which the binding ruptures, normally depends on the contour length of the molecules involved, on how they were immobilized, and on the location of their binding sites. 3) The adhesion probability is the ratio between the number of approach-retract cycles with adhesion and the total number of cycles. It generally depends on the molecular surface coverage, the buffer composition and the kinetics of the binding (Dammer et al., 1996; Hinterdorfer et al., 1996). 4) The area between the approach and retract branch of a force-distance curve theoretically corresponds to the energy dissipated during the stretching and rupture process. However, a straightforward analysis is difficult, because of different intra- and intermolecular contributions of entropic and energetic effects to this energy.

Most extensive studies have been done on the biotin - avidin family, where also a correlation between the measured rupture forces and the binding enthalpy was found (Moy et al., 1994, Chilkoti et al., 1995) and the measured forces could be confirmed by a molecular mechanic simulation (Grubmüller et al., 1996).

Furthermore, the shape of the hysteresis curve of an approach-retract cycle can give information about mechanical properties such as the elasticity of the biomolecules. In Fig. 2, three different force-distance curves are given. All were recorded in liquids (see preparation). The top curve of Fig. 2 represents a measurement between a gold tip and a glass surface functionalized with mercaptosilane. Due to the high affinity between the gold covered sensor and the SH-groups on the flat surface a strong binding of multiple $\mathrm{Au}-\mathrm{SH}$ bonds can form, resulting in considerable adhesion forces of about $2.5 \mathrm{nN}$. Furthermore, the very pronounced and linear force-distance dependence in the retracting branch of the curve indicate a very stiff and short range interaction. In Fig. 2b, a typical measurement between an avidin functionalized surface and a biotin functionalized tip is given. The system of the globular protein and the small ligand exhibit two distinct differences to the curve described above. First the measured adhesion forces are considerably smaller (here: $120 \mathrm{pN}$ ) and secondly, the shape of the curve clearly deviates from a straight line, indicating contribution from the mechanical response of the system to the external applied force.

As a third example, Fig. 2c represents an interaction measurement between a sensor and a surface both functionalized by cell adhesion molecules (MAF) as already introduced above and visualized in Fig. 1c and 1d. The MAF molecules are thought to interact via their long carbohydrate rich arms, which have multiple binding sites for one another and a length of about $150 \mathrm{~nm}$ (see Fig. 1c). The retarded shape of the retracting branch of the curve relates to the phenomenon of two interacting chain-like molecules such as carbohydrates, DNA or polymer systems. Thereby, the force acting on the sensor, first has to be established upon stretching the molecules.

Depending on the force regime, two fundamental phenomena contribute to this nonlinear response: the low forces regime (below some ten $\mathrm{pN}$ ) is dominated by entropic effects and the cantilever has to work against a random thermal motion, which tends to curl the molecule at room temperature. At higher forces additional energetic contributions from the elasticity of single chain segments have to be considered. Recently, stretching experiments with DNA as a model system showed entropic as well as energetic elastic phenomena such as structural phase transitions (Smith et al., 1996; Cluzel et al., 1996).

The entropic elasticity of a chain-like molecule can be modelled with an inverse Langevin function, deduced from a freely jointed chain model (FJC) of orientationally independent Kuhn segments (Bueche, 1979), or by empirical or interpolation formulas (Reese and Zimm, 1990; Bustamante et al., 1994). The energetic contribution, which describes the elastic property of single segments of chain-like molecules, can be taken into account by adding a linear term containing a stretch modulus or a molecular spring constant to the entropic formulas (Smith et al., 1996).

Upon analyzing the curve in Fig. 2c, we get the best correspondence to the experimental data by simulating the elastic response of MAF arms within the framework of pure entropic elasticity. The curve in Fig. 3 was fitted with an interpolation formula describing the entropic elasticity in the small and large force regimes (Bustamante et al., 1994): 


$$
F(x)=\frac{k T}{A}\left(\frac{1}{4}\left(1-\frac{x}{L}\right)^{-2}-\frac{1}{4}+\frac{x}{L}\right)
$$

where $\mathrm{k}$ and $\mathrm{T}$ denote Boltzman constant and temperature. This model has two free parameters: 1) the persistence length $\boldsymbol{A}$, which describes the length of the chain segments and 2) the molecular contour length $\boldsymbol{L}$ of the chain (Smith et al., 1992; Bustamante et al., 1994). For the case of the thin carbohydrate rich arm of the MAF molecules, we obtain a Kuhn length of $\boldsymbol{b}=0.94 \mathrm{~nm}$ (which corresponds to twice the persistance length $\boldsymbol{A}$ ) and a molecular chain length of $220 \mathrm{~nm}$ (figure 3). Herewith, considering a MAF arm length of $\sim 150 \mathrm{~nm}$ (see also figure 1c), it can be concluded that the MAF-MAF-interaction binding sites are not (only) located at the rear ending of the arms, but also situated along them.

This is a first approach to model the shape of an AFM force-distance curve of biomolecules by a general model of molecular elasticity and by neglecting the complicated internal structure of the molecule and other parameters such as charge distribution in and around the molecule.

\section{SUMMARY AND OUTLOOK}

We showed current applications of AFM in biology like imaging of ssRNA, IgGs and goldlabelled cell adhesion molecules. The AFM allows imaging of these systems with a resolution of about $5 \mathrm{~nm}$, and can be used to determine the activity of molecules or to identify binding sites on molecules by an easy sample preparation. By imaging gold-labelled molecules, the $5 \mathrm{~nm}$ gold beads seem to be a good compromise between lateral resolution and distinguishing the beads from molecular structures. Identification of smaller beads by electrostatic or phase contrast AFM could be possible in the future.

Additionally, AFM can measure forces between biomolecules, and give information about their binding, molecular structure and elasticity. Recent experiments in our lab on sugar protein interactions aim to investigate dynamic properties of single molecules such as their rate constants and the force dependence of the lifetime of a binding by varying the approachretract velocity of the AFM sensor. It seems, that especially in case of systems of surface bound molecules, which have on- and off rates in the timescale of AFM force-distance measurements (msec to minutes), the AFM can give new insights in the binding properties of these molecules.

We acknowledge important discussions with Alfredo E. Bruno, Max Burger, Hans-Joachim Güntherodt, Ernst Meyer and Angelika Muscate and technical support from Loris Scandella and Evelyn Schürmann.

\section{REFERENCES}

Bueche, F. (1979) In Physical properties of polymers, Robert E. Krieger Publishing Company, New York.

Bustamante, C., Marko, J.F., Siggia, E.D. and Smith, S. (1994) Entropic elasticity of $\lambda$ phage DNA, Science 265, 1599-1600.

Bustamante, C., and Keller, D. (1995) Scanning force microscopy in biology, Physics Today December, 32-38.

Chilkoti, A., Boland, Th., Ratner, B.D., and Stayton, P.S. (1995) The relationship between ligand-binding thermodynamics and protein-ligand interaction forces measured by atomic force microscopy, Biophys. J. 69, 2125-2130

Cluzel, P., Lebrun, A., Heller, Ch., Lavery, R., Viovy, J.-L., Chatenay, D. and Caron, F. (1996) DNA: an extensible molecule, Science 271, 792-794.

Dammer, U., Anselmetti, D., Dreier, M., Hegner, M., Huber, W., Hurst, J., Misevic, G., and Güntherodt, H.-J. (1995a) Measuring molecular adhesion with force microscopy, In Forces in Scanning Probe Methods, NATO ASI Series E 286. H.-J. Güntherodt, D. Anselmetti and E. Meyer, editors. Kluwer Academic Press, Dordrecht, 625-631.

Dammer, U., Popescu, O., Wagner, P. Anselmetti, D., Güntherodt, H.-J., and Misevic, G.N. (1995b) Binding strength between cell adhesion proteoglycans measured by atomic force microscopy, Science 267, 1173-1175.

Dammer, U., Hegner M., Anselmetti, D., Wagner, P., Dreier, M., Huber, W., and Güntherodt, H.-J. (1996) Specific Antigen/Antibody Interactions Measured by Force Microscopy, Biophys. J. 70, 2437-2441. 
Evans, E., Berk, D. and Leung, A. (1991) Detachment of agglutinin-bonded red blood cells, Biophys. J. 59, 838-848.

Florin, E.-L., Moy, V.T. and Gaub, H.E., (1994) Adhesion forces between individual ligand- receptor pairs, Science 264, 415417.

Florin, E-L., Rief, M., Lehmann, H., Ludwig, M., Dornmair, C., Moy, V.T. and Gaub, H.E. (1995) Sensing specific molecular interactions with the atomic force microscope, Biosensors \& Bioelectronics 10, 895-901.

Grubmüller, H., Heymann, B. and Tavan, P. (1996) Ligand binding: molecular mechanics calculation of the streptavidinbiotin rupture force, Science 271, 997999.

Hansma, H.G., Sinsheimer, R.L., Groppe, J., Bruice, T.C., Elings, V., Gurley, G., Bezanilla, M., Mastrangelo, I.A., Hough, P.V.C., and Hansma, P.K. (1993) Recent advances in atomic force microscopy of DNA, Scanning 15, 296-299.

Hansma, H.G., and Hoh, J., (1994) Biomolecular imaging with the atomic force microscope, Ann. Rev. Biophys. Biomol. Struct. 23, 115-139.

Hinterdorfer, P., Baumgartner, W., Gruber, H.J., Schilcher, K. and Schindler, H. (1996) Detection and localization of individual antibody-antigen recognition events by atomic force microscopy, Proc. Natl. Acad. Sci. USA 93, 3477-3481.

Israelachvili, J.N., (1992) In Intermolecular and Surface Forces, Academic Press, London.

Karrasch, S., Dolder, M., Schabert, F., Ramsden, J., and Engel, A. (1993) Covalent binding of biological samples to solid supports for scanning probe microscopy in buffer solution, Biophys. $J$. 65, 2437-2446.

Leckband, D. (1995) The surface force apparatus - a tool for probing molecular protein interactions, Nature 376, 617-618.

Lee, G.U., Chrisey, L.A., and Colton, R.J., (1994a) Direct measurement of the forces between complementary strands of DNA, Science 266, 771-773.

Lee, G.U., Kidwell, D.A., and Colton, R.J., (1994b) Sensing discrete streptavidinbiotin interactions with atomic force microscopy, Langmuir 10, 354-357.
Meyer, E., Heinzelmann, H., Grütter, P., Jung, Th., Weisskopf, Th., Hidber, H-R., Lapka, R., Rudin, H., and Güntherodt, H.-J. (1988) Comparative study of lithium fluoride and graphite by atomic force microscopy (AFM), Journal of Microscopy 152, 269-280.

Misevic, G.N. and Burger, M.M. (1993) Carbohydrate-carbohydrate interactions of novel acidic glycan can mediate sponge cell adhesion, J. Biol. Chem. 268, 49224929.

Moy, V.T., Florin, E.-L. and Gaub, H.E. (1994) Intermolecular forces and energies between ligands and receptors, Science 266, 257-259.

Neumeister, J.M., and Ducker, W.A. (1994) Lateral, normal, and longitudinal spring constants of atomic force microscopy cantilevers, Rev. Sci. Instrum. 65, 25272531.

Reese, H.R. and Zimm, B.H., (1990) Fracture of polymer chains in extensional flow: Experiments with DNA, and a moleculardynamics simulation, J. Chem. Phys. 92, 2650-2662.

Schabert, F.A., Henn, Ch., and Engel, A. (1995) Native Escherichia coli OmpF porin surfaces probed by atomic force microscopy, Science 268, 92-94.

Smith, S.B., Finzi, L. and Bustamante, C. (1992) Direct mechanical measurements of the elasticity of single DNA molecules by using magnetic beads, Science 258, 11221126.

Smith, S.B., Cui, Y., and Bustamante, C. (1996) Overstretching B-DNA: the elastic response of individual double-stranded and single-stranded DNA molecules, Science 271, 795-799.

Thundat, T., Allison, D.P., Warmack, R.J., Brown, G.M., Jacobson, K.B., Schrick, J.J., and Ferrell, T.L. (1992) Atomic force microscopy of DNA on mica and chemically modified mica, Scanning Microscopy 6, 911-918.

Svoboda, K., Schmitt, Ch.F., Schnapp, B.J., and Block, S.M. (1993) Direct observation of kinesin stepping by optical trapping interferometry, Nature 365, 721-727

Wagner, P., Hegner, M., Kernen, P., Zaugg, F., and Semenza, G. (1996) Covalent immobilization of native biomolecules onto 
$\mathrm{Au}(111)$ via N-hydroxysuccinimide ester functionalized self-assembled monolayers for scanning probe microscopy, Biophys. J. 70, 2052-2066.

Weisenhorn, A.L., Maivald, P., Butt, H.-J., and Hansma, P.K. (1992) Measuring adhesion, attraction, and repulsion between surfaces in liquids with an atomic-force microscope, Phys. Rev. B 45, 1122611232. 
Fritz et al.
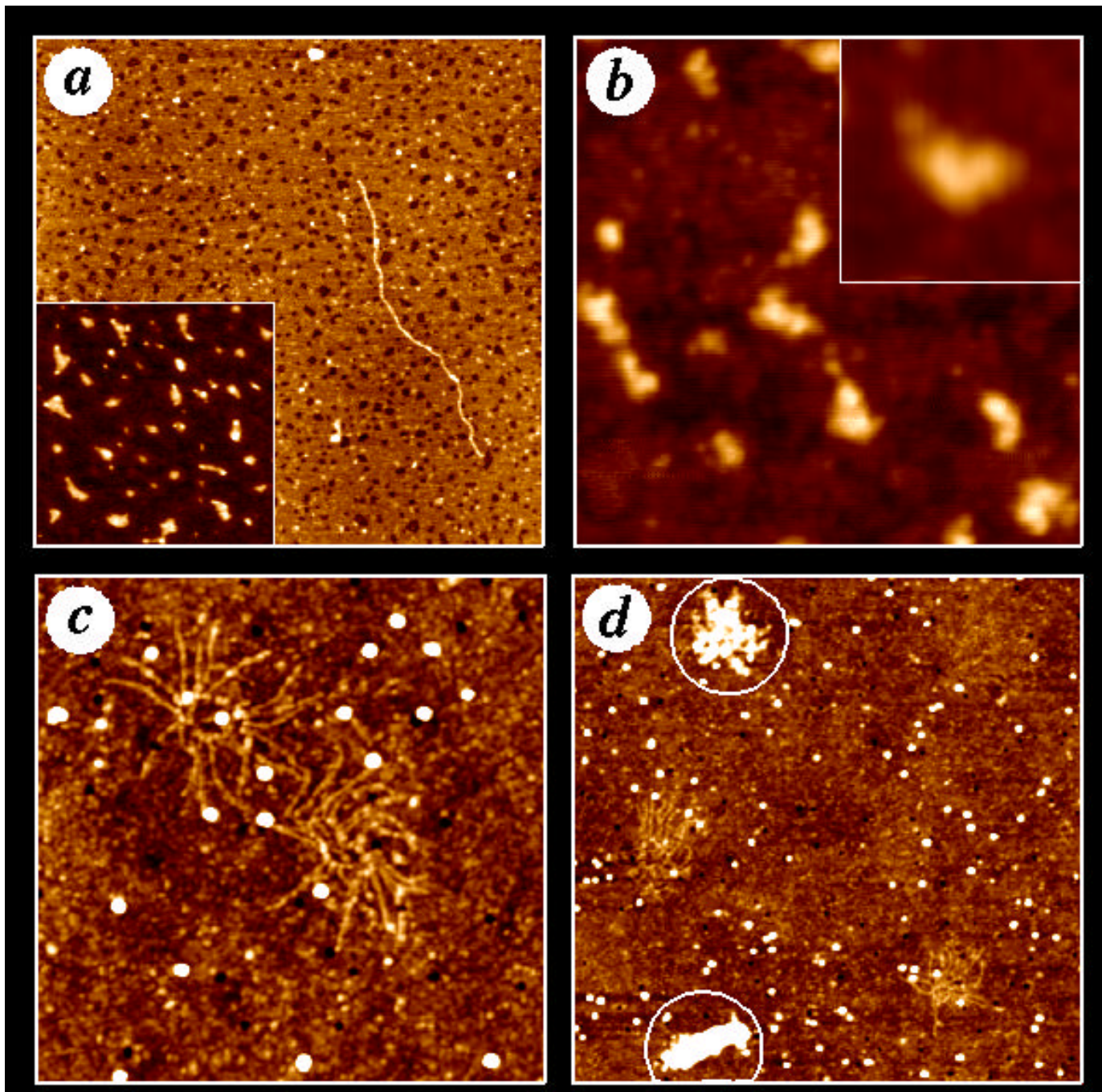

FIG. 1. AFM images of biomolecules in air: (a) ssRNA molecules immobilized on a silanized glass surface. The inset image shows ssRNA molecules in a conformational state exhibiting a globular structure, whereas the chain-like ssRNA molecule in the full image was denatured with formamide $(2 \mu \mathrm{m} \times 2 \mu \mathrm{m}$, inset: $1 \mu \mathrm{m} \times 1 \mu \mathrm{m})$. (b) Random oriented IgGantibodies immobilized on mica. The characteristic heart-shaped structure can clearly be identified $(300 \mathrm{~nm} \times 300 \mathrm{~nm}$, inset:

$50 \mathrm{~nm} \times 50 \mathrm{~nm}) .(\mathrm{c}) \&(\mathrm{~d})$ Immunogold labelling experiment with cell adhesion molecules (MAF) immobilized on a cover slip. The molecules were labelled with $5 \mathrm{~nm}-\mathrm{Au}$ beads (bright spots) via antiMAF directed and secondary antibodies. The dark spots are characteristic holes in the glass surface. In (d) two classes of MAF-molecules with high (see circles) and low affinity for $\mathrm{Ab}$ binding can clearly be identified (c) $1 \mu \mathrm{m} \times 1 \mu \mathrm{m}$; (d) $2 \mu \mathrm{m}$ $\times 2 \mu \mathrm{m}$. 

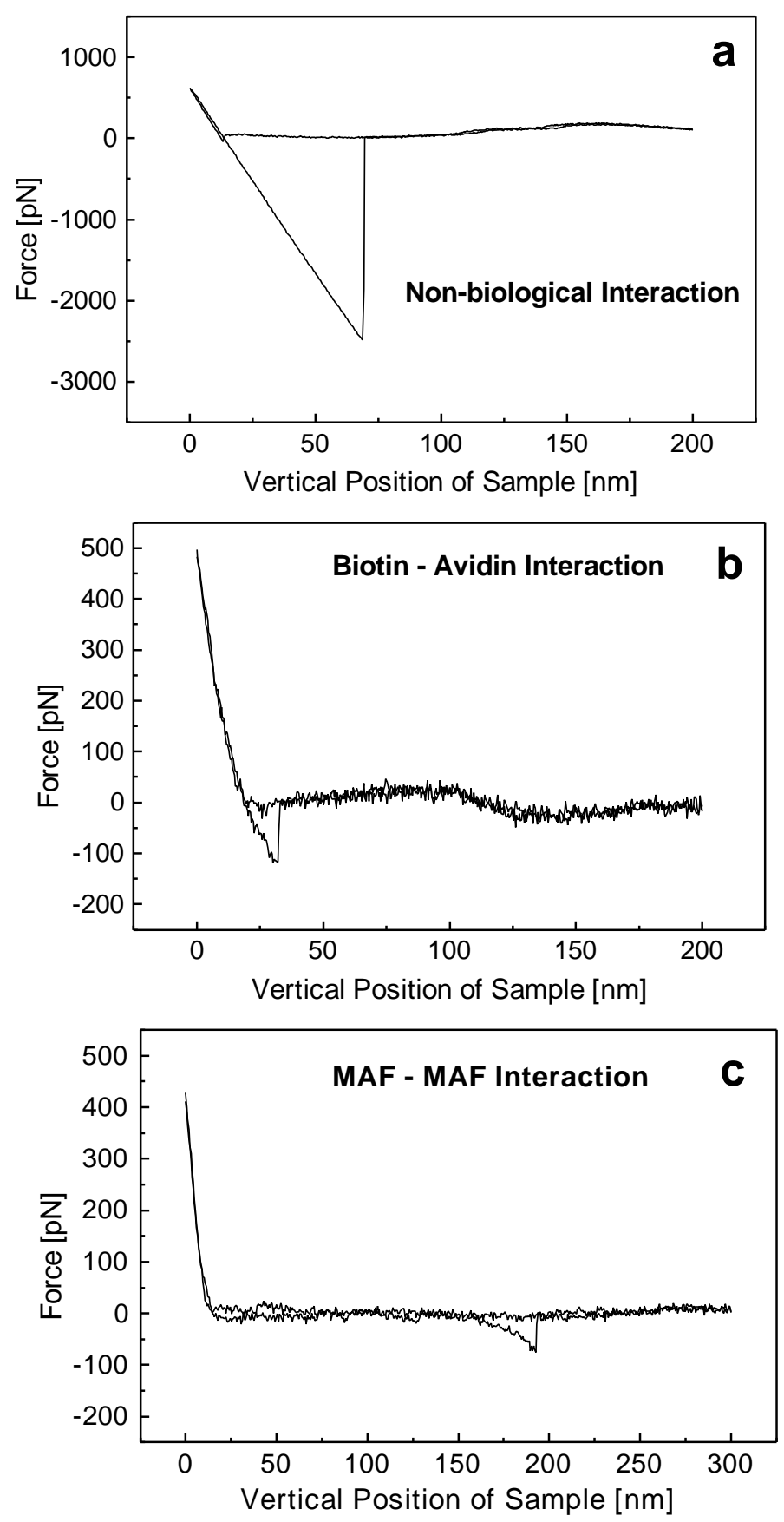

FIG. 2. Three different raw data AFM forcedistance curves obtained with functionalized AFM force sensors. The force acting on the sensor tip is plotted versus the vertical position of the sample surface. (a) Interaction between a gold covered tip and a mercapto-silanized glass surface in water. (b) Interaction between a biotin functionalized tip and an avidin functionalized glass surface in PBS buffer (The small wave-like baseline shift in the right part of the curve is due to interference artifacts of the laser beam detection of the AFM). (c) Interaction between cell adhesion molecules (MAF) immobilized on AFM tip and surface in $\mathrm{Ca}^{2+}$-containing Tris-buffered seawater. 
Fritz et al.

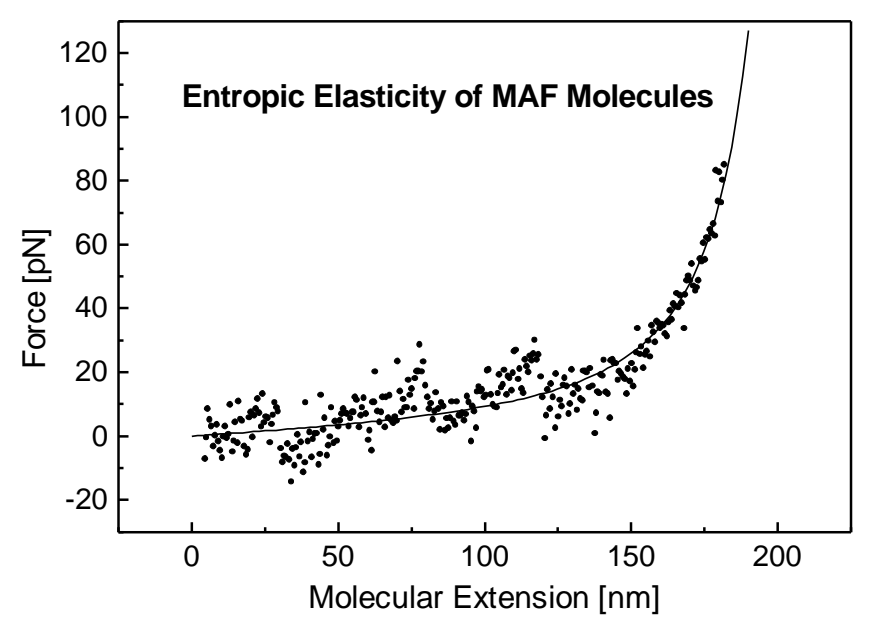

FIG. 3. Experimental data (from Fig. 2c) and elasticity fit of an AFM interaction experiment between two cell adhesion molecules (MAF). In order to extract the extension of the molecules, the vertical sample position of the forcedistance curve was corrected by the deflection of the AFM force sensor. The elastic response of two interconnecting MAF arms on an external force was fitted with a purely entropic model, yielding a Kuhn segment length $\boldsymbol{b}$ of $0.94 \mathrm{~nm}$ and a molecular contour length $\boldsymbol{L}$ of $220 \mathrm{~nm}$. 